\title{
Ocorrência de Cryptosporidium spp. em dois centros de treinamento de equinos de Curitiba, Paraná
}

\section{Occurrence of Cryptosporidium spp. in two centers of training horses in Curitiba, Paraná}

\author{
Keila Youko FUJII'; João Ricardo DITTRICH ${ }^{1}$; \\ Edilene Alcântara de CASTRO²; Jonatas Campos de ALMEIDA ${ }^{3}$ \\ ${ }^{1}$ Universidade Federal do Paraná, Setor de Ciências Agrárias, Departamento de Zootecnia, Curitiba - PR, Brasil \\ ${ }^{2}$ Universidade Federal do Paraná, Setor de Ciências Biológicas, Departamento de Patologia Básica, Curitiba - PR, Brasil \\ ${ }^{3}$ Universidade Federal de Londrina, Setor de Ciências Agrárias, \\ Departamento de Medicina Veterinária Preventiva, Londrina - PR, Brasil
}

\begin{abstract}
Resumo
O presente trabalho investigou a ocorrência de parasitismo por Cryptosporidium spp. em equinos alojados em dois centros de treinamento de equinos localizados no município de Curitiba, Paraná. Foram examinados 108 cavalos, sendo 48 procedentes do Centro de Treinamento 1 (CT1) e 60 do Centro de Treinamento 2 (CT2). As coletas de amostras de fezes foram realizadas no período de outubro de 2010 a janeiro de 2011. A metodologia utilizada para a confirmação da presença de oocistos de Cryptosporidium spp. foi a técnica de Ziehl-Neelsen modificada. A ocorrência encontrada foi de $18,52 \%$ para o total de animais examinados. Houve diferença estatística significativa ( $p>0,05)$ quando comparadas as prevalências encontradas nos dois centros de treinamento, sendo no CT1 de 4,16\% e no CT2 de $30 \%$. Não houve associação entre a prevalência e a idade, $o$ sexo e raça $(p>0,05)$.
\end{abstract}

Palavras-chaves: Ambiente. Cavalos. Oocistos.

\begin{abstract}
This study investigated the occurrence of parasitism by Cryptosporidium spp. in horses housed in two training centers located in Curitiba, Paraná. A total of 108 horses were examined, 48 from the Training Center 1 (CT1) and 60 of the Training Centre 2 (CT2). Collection of stool specimens occurred from October 2010 to January 2011. The methodology used to confirm the presence of Cryptosporidium spp. was the Ziehl-Neelsen modified. The occurrence found was $18.52 \%$ for the total animals examined. There was a statistically significant difference $(p>0.05)$ comparing prevalence rates in the two training centers: $4.16 \%$ in CT1 and 30\% in CT2. There was no association between prevalence and the age, sex and race $(\mathrm{p}>0.05)$ of the horses.
\end{abstract}

Keywords: Environment. Horses. Oocysts.

\section{Introdução}

O protozoário do gênero Cryptosporidium está ganhando cada vez mais atenção como um agente patogênico do homem e dos animais, principalmente devido a surtos de criptosporidiose por veiculação hídrica associados ao acesso de seres humanos a reservatórios de água contaminada (SMITH; NICHOLS, 2010).

Devido à sua estreita relação com a população de baixo poder aquisitivo, precárias condições de saneamento básico e da qualidade da água consumida, esse parasito foi incluído na lista de doenças negligenciadas da Organização Mundial da Saúde (SAVIOLI; SMITH; THOMPSON, 2006; ASSIS et al., 2013). O impacto sanitário e econômico dessa zoonose ainda é indeterminado, principalmente porque alguns representantes do gênero Cryptosporidium não são espécieespecíficos e apresentam risco de transmissão para outros animais e para humanos (PONCE GORDO; HERRERA; CASTRO, 2002).

Atualmente, são reconhecidas 26 espécies de Cryptosporidium, das quais nove podem infectar humanos

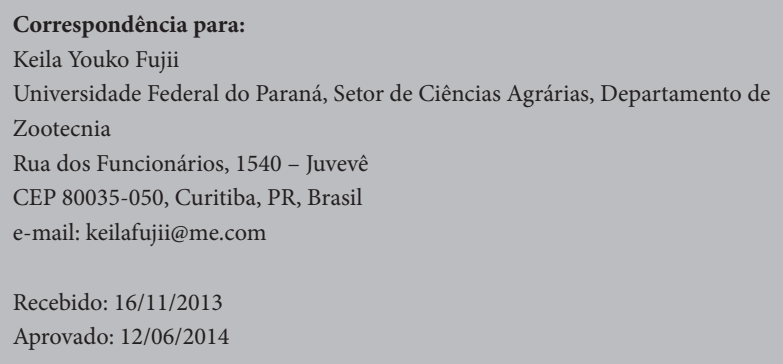


(ASSIS et al., 2013; SHARMA et al., 2013). As formas de transmissão já documentadas são de animais para o ser humano, de pessoa para pessoa e de animal para animal, por meio da ingestão de alimentos ou do uso de água destinada ao lazer contaminados direta ou indiretamente por resíduos fecais que contenham oocistos esporulados do parasita (MAIKAI; BABAONOJA; ELISHA, 2013; WIDMER et al., 2012).

A importância da criptosporidiose em animais de produção é traduzida não só pelo potencial zoonótico de algumas espécies como também pela perda econômica que a infecção promove (OLSON et al., 2004). Apesar de o protozoário Cryptosporidium spp. ter ocorrência cosmopolita, a incidência do agente em cavalos é pouco documentada e pode variar com o método de detecção utilizado e a população estudada (MAIR; COHEN; PEARSON, 2009).

Entre os métodos laboratoriais disponíveis para o diagnóstico do parasitismo pelo Cryptosporidium spp., tem sido observada a concordância dos resultados obtidos com a técnica de coloração de Ziehl-Neelsen e os ensaios imunoenzimáticos (MAJEWSKA et al., 2004).

Entre os inquéritos já realizados no Brasil investigando o parasitismo de equinos pelo Cryptosporidium spp., Gomes et al. (2008) e Toscan et al. (2010), empregando a Técnica de Faust modificada, encontraram, respectivamente, ocorrências de $75 \%$ e $80,8 \%$ de animais positivos no Jockey Clube de Santa Maria, RS, e ressaltaram que as altas ocorrências encontradas poderiam estar relacionadas a uma possível contaminação hídrica existente no local.

O presente trabalho investigou a ocorrência de Cryptosporidium spp. em dois centros de treinamento de equinos no município de Curitiba, Paraná.

\section{Material e Método}

O trabalho foi realizado no período de outubro de 2010 a janeiro de 2011 em dois centros de treinamento de equinos localizados no município de Curitiba, Paraná. Foram examinados 108 animais, dos quais 48 do Centro de Treinamento 1 (CT1) e 60 do Centro de
Treinamento 2 (CT2). Dos 48 animais do CT1, 35 eram machos e 13 fêmeas, sendo 41 de Raças Puras (RP) e sete Sem Raça Definida (SRD). Dos 60 animais do CT2, 48 eram machos e 12 fêmeas, 45 eram animais SRD e 15 eram RP. A idade dos animais variou entre três e 21 anos. Os animais utilizados não apresentavam qualquer alteração clínica sugestiva de criptosporidiose.

A base de alimentação dos animais do CT2 era constituída por ração concentrada (14\% de proteína bruta) e aveia branca não achatada. Eram fornecidos diariamente quatro quilos por dia dessa mistura, por animal, divididos em duas vezes (manhã e tarde) em cochos de cimento fixados no interior da cocheira. Também eram fornecidos feno de alfafa, capim verde e sal mineral. No CT1, cada animal recebia uma dieta específica dependendo do proprietário. As principais diferenças nos CTs ocorriam nas proporções e na qualidade da ração concentrada utilizada, que dependia das atividades atléticas dos animais. As camas, em ambos os centros de treinamento, eram constituídas por maravalha. As fezes eram removidas duas vezes ao dia no CT1 e uma vez ao dia no CT2, sendo depositadas próximo às cocheiras até a sua retirada. A cada oito dias eram recolocados aproximadamente 50 $\mathrm{kg}$ de maravalha para substituir a cama que era totalmente removida. A água utilizada nos dois locais é tratada pela Companhia de Saneamento do Paraná (Sanepar), com conexão de fornecimento individual.

As amostras foram coletadas diretamente do bolo fecal imediatamente após a excreção, coletando-se apenas a porção superior. Essas amostras foram identificadas, armazenadas em caixas isotérmicas contendo gelo reciclável e transportadas até o laboratório para serem examinadas.

O diagnóstico de oocistos de Cryptosporidium spp. foi efetuado com a técnica de esfregaço de fezes em lâmina, corado com o método de Ziehl-Neelsen modificado (HENRIKSEN; POHLENZ, 1981).

Foram comparadas as ocorrências de oocistos Cryptosporidium spp. nos dois centros de treinamento e as variáveis idade, sexo e raça. 
Para a análise estatística foi empregada a análise de variância executada com o programa estatístico SISVAR 5.3 (FERREIRA, 2010). A ocorrência do parasita nas faixas etárias, sexo e raça foi analisada pelo teste exato de Fischer ao nível de significância de 5\%.

\section{Resultados e Discussão}

A ocorrência observada foi de 18,52\% (20/108) para o total de animais examinados e os valores observados no CT1 $(4,16 \%, 2 / 48)$ e CT2 $(30 \%, 18 / 60)$ apresentaram diferença significante entre si $(\mathrm{p}<0,05)$.

Valores semelhantes aos encontrados no CT1 foram verificados por Majewska et al. (2004) em um centro de equitação na Polônia, com ocorrência de 3,5\%, utilizando a técnica de coloração Ziehl-Neelsen e o ensaio imunoenzimático. Marques (2010), examinando 90 amostras fecais de equinos de Porto Alegre, RS com a coloração de Ziehl-Neelsen, observou oocistos de Cryptosporidium spp. em 27,87\% (25/90) dos animais. Os equinos da Corporação Militar do Rio de Janeiro, RJ, apresentaram uma prevalência de 0,65\%, considerada baixa (SOUZA et al., 2009). Já ausência de oocistos do protozoário foi relatada por Johnson et al. (1997) em 91 equinos da Califórnia, USA, e por Morais et al. (2008) em Uberlândia, MG, em uma propriedade com 31 animais.

No CT1, onde foi observada baixa ocorrência de animais positivos para Cryptosporidium spp., os animais passavam menos tempo confinados, saindo de suas baias para treinamentos e competições, o que difere do CT2 onde os equinos ficavam a maior parte do tempo em confinamento. A limpeza diária das baias era efetuada duas vezes no CT1 e uma vez no CT2, o que poderia explicar a maior proporção de animais positivos encontrada no CT2, pois o tipo de manejo adotado favorecia o acúmulo de fezes no ambiente com maior oportunidade para a contaminação local e a disseminação dos oocistos para outras baias pelos próprios tratadores. Bomfim et al. (2005) e Causape et al. (2002) citam que o ambiente de confinamento favorece à infecção pelo protozoário devido ao maior tempo de permanência nas instalações e pelo aumento de dejetos e umidade no local. O tipo de piso, a higiene, o uso de desinfetante, a procedência da água consumida pelos animais, o tipo de alimentação e a forma de armazenamento da mesma podem ser fatores de risco para a infecção por Cryptosporidium spp. (HAMNES; GJERDE; ROBERTSON, 2006; BROOK et al., 2008).

Apesar de o fator idade ter se mostrado como elemento de risco para infecção por Cryptosporidium spp. em potros com menos de dois meses (INÁCIO et al., 2012), no presente trabalho não foi constatada associação entre a idade e a ocorrência do protozoário ( $\mathrm{p}>0,05)$. Possivelmente, isso se deve ao pequeno número de animais jovens examinados (12/108). Também não foi observada associação entre a presença de oocistos nas fezes e o sexo dos animais. Uma vez que a porcentagem de machos examinados foi maior $(75 \%$, $81 / 108)$ que a das fêmeas $(25 \%, 27 / 108)$, esta grande diferença pode ter influenciado o resultado obtido, apesar de outros autores também não terem verificado tal associação (GOMES et al., 2008). O mesmo também foi observado com relação à ocorrência encontrada entre animais RP $(25 \%, 5 / 20)$ e SRD $(75 \%$, $15 / 20)$, em que não houve diferença estatística entre as populações. Entretanto, Toscan et al. (2010) observaram maior prevalência em equinos da raça PSI $(80,8 \%)$ quando comparados aos SRD (38,5\%).

\section{Conclusão}

Os resultados obtidos no presente trabalho identificaram diferenças nas ocorrências de equinos parasitados por Cryptosporidium spp. em dois centros hípicos localizados no município de Curitiba, estado do Paraná, Brasil, atribuídas possivelmente à diferença de manejo, com destaque para a frequência de limpeza adotada. 


\section{Referências}

ASSIS, D. C.; RESENDE, D. V.; SANTOS, M. C.; CORREIA, D.; OLIVEIRA-SILVA, M. B. Prevalence and genetic characterization of Cryptosporidium spp. and Cystoisospora belli in HIV-infected patients. Revista do Instituto de Medicina Tropical de São Paulo, v. 55, n. 3, p. 149-154, 2013. Disponível em: <http://www.scielo.br/ scielo.php?script=sci_arttext\&pid=S0036-46652013000300149>. Acesso em: 15 out. 2013. doi: http://dx.doi.org/10.1590/S003646652013000300002.

BOMFIM, T. C. B.; HUBER, F.; GOMES, R. S.; ALVES, L. L. Natural infection by Giardia sp. and Cryptosporidium sp. in dairy goats, associated with possible risk factors of the studied properties. Veterinary Parasitology, v. 134, n. 1-2, p. 9-13, 2005. Disponível em: <http://www.sciencedirect.com/science/article/ pii/S0304401705003468>. Acesso em: 24 set. 2013. doi: http:// dx.doi.org/10.1016/j.vetpar.2005.05.067.

BROOK, E. J.; CHRISTLEY, R. M.; FRENCH, N. P.; HART, C. A. Detection of Cryptosporidium oocysts in fresh and frozen catlle faeces: comparison of three methods. Letters in Applied Microbiology, v. 46, n. 1, p. 26-31, 2008. Disponível em: <http:// onlinelibrary.wiley.com/doi/10.1111/j.1472-765X.2007.02257.x/ abstract $>$. Acesso em: 7 set. 2013. doi: http://dx.doi.org/10.1111/ j.1472-765X.2007.02257.x.

CAUSAPE, A. C.; QUÍlEZ, J.; SÁNCHEZ-ACEDO, C.; DEL CACHO, E.; LÓPEZ-BERNAD, F. Prevalence and analysis of potential risk factors for Cryptosporidium parvum infection in lambs in Zaragoza (northeastern Spain). Veterinary Parasitology, v. 104 n. 4, p. 287-298, 2002. Disponível em: <http://www.sciencedirect. com/science/article/pii/S0304401701006392 > . Acesso em: 24 set. 2013. doi: http://dx.doi.org/10.1016/S0304-4017(01)00639-2.

FERREIRA, D. F. SISVAR: Sistema de Análise de Variância. Versão 5.3. Lavras: UFLA, 2010.

GOMES, A. D.; BARRETTA, C.; ZIEGLER, D. P.; SAUSEN, L.; STOEVER, N.; SANGIONI, L. A.; VOGEL, F. S. F.; MONTEIRO, S. G.; ZANELLA, A. Prevalência de Cryptosporidium spp e Giardia sp em eqüinos estabulados no Jockey Club de Santa Maria - RS, Brasil. Ciência Rural, v. 38, n. 9, p. 2662-2665, 2008. Disponível em: <http://www.scielo.br/scielo.php?script=sci_artt ext\&pid=S0103-84782008000900045>. Acesso em: 19 out. 2013. doi: http://dx.doi.org/10.1590/S0103-84782008005000012.

HAMNES, I. S.; GJERDE, B.; ROBERTSON, L. Prevalence of Giardia and Cryptosporidium in dairy calves in three areas of Norway. Veterinary Parasitology, v. 140, n. 3-4, p. 204-216, 2006. Disponível em: <http://www.sciencedirect.com/science/article/ pii/S0304401706002238>. Acesso em: 14 out. 2013. doi: http:// dx.doi.org/10.1016/j.vetpar.2006.03.024.

HENRIKSEN, S. A.; POHLENZ, J. F. L. Staining of Cryptosporidia by a modified Ziehl-Neelsen technique. Acta Veterinaria Scandinavica, v. 22, n. 3-4, p. 594-596, 1981.

INÁCIO, S. V.; BRITO, R. L. L.; ZUCATTO, A. S.; COELHO, W. M. D.; AQUINO, M. C. C.; AGUIRRE, A. A. R.; PERRI, S. H. V.; MEIRELES, M. V.; BRESCIANI, K. D. S. Cryptosporidium spp. infection in mares and foals of the northwest region of São Paulo State, Brazil. Revista Brasileira de Parasitologia Veterinária, v. 21, n. 4, p. 355-358, 2012. Disponível em: <http://www.scielo.br/ scielo.php?pid=S1984-29612012000400003\&script=sci_arttext $>$. Acesso em: 13 nov. 2013. doi: http://dx.doi.org/10.1590/S198429612012005000003.

JOHNSON, E.; ATWILL, E. R.; FILKINS, M. E.; KALUSH, J. The prevalence of shedding of Cryptosporidium and Giardia spp. based on a single fecal sample collection from each of 91 horses used for backcountry recreation. Journal of Veterinary Diagnostic Investigation, v. 9, n. 1, p. 56-60, 1997.

MAIKAI, B. V.;BABA-ONOJA,E. B. T.;ELISHA, I. A. Contamination of raw vegetables with Cryptosporidium oocysts in markets within Zaria metropolis, Kaduna State, Nigeria. Food Control, v. 31, n. 1, p. 45-48, 2013. Disponível em: <http://www.sciencedirect.com/ science/article/pii/S0956713512005324>. Acesso em: 14 set. 2013. doi: http://dx.doi.org/10.1016/j.foodcont.2012.09.032.
MAIR, T. S.; COHEN, N. D.; PEARSON, G. R. Cryptosporidiosis. In: MAIR, T. S.; COHEN, N. D.; PEARSON, G. R. Infectious diseases of the horses. Mereworth: Bell Equine Veterinary Clinic, 2009. p. 347-353.

MAJEWSKA, A. C.; SOLARCZK, P.; TAMANG, L.; GRACZK, T. K. Equine Cryptosporidium parvum infections in western Poland. Parasitology Research, v. 93, n. 4, p. 274-278, 2004. Disponível em: <http://link.springer.com/article/10.1007\%2Fs00436-0041111-y>. Acesso em: 6 set. 2013. doi: http://dx.doi.org/10.1007/ s00436-004-1111-y.

MARQUES, S. M. T. Cryptosporidiosis in horses of urban areas of Porto Alegre, Rio Grande do Sul, Southern Brazil. Journal of Equine Veterinary Science, v. 30, n. 7, p. 356-358, 2010. Disponível em: <http://www.sciencedirect.com/science/article/ pii/S0737080610002261>. Acesso em: 15 out. 2013. doi: http:// dx.doi.org/10.1016/j.jevs.2010.05.006.

MORAIS, T. M.; MUNDIM, M. J. S.; ARANTES, V. M.; COSTA, F. C.; ROSA, L. A. G.; FARIA, S. M.; CURY, M. C. Prevalência e fatores de risco relacionados à infecção por Giardia duodenalis e Cryptosporidium spp. em diferentes espécies de animais. In: ENCONTRO INTERNO, 8., SEMINÁRIO DE INICIAÇÃO CIENTÍFICA, 12., 2008, Uberlândia. Anais... Uberlândia: Universidade Federal de Uberlândia, 2008.

OLSON,M.E.;O'HANDLEY,R.M.;RALSTON,B.J.;McALLISTER, T. A.; THOMPSON, R. C. A. Update on Cryptosporidium and Giardia infections in cattle. Trends in Parasitology, v. 20, n. 4, p. 185-191, 2004. Disponível em: <http://www.sciencedirect.com/ science/article/pii/S1471492204000303>. Acesso em: 27 ago. 2013. doi: http://dx.doi.org/10.1016/j.pt.2004.01.015.

PONCE GORDO, F.; HERRERA, S.; CASTRO, A. T. Parasites from ostriches (Struthio camelus) and rheas (Rhea Americana) in Europe. Veterinary Parasitology, v. 107, n. 1-2, p. 137-160, 2002. Disponível em: <http://www.sciencedirect.com/science/article/ pii/S0304401702001048>. Acesso em: 4 nov. 2013. doi: http:// dx.doi.org/10.1016/S0304-4017(02)00104-8.

SAVIOLI, L.; SMITH, H.; THOMPSON, A. Giardia and Cryptosporidium join the 'neglected diseases initiative'. Trends in Parasitology, v. 22, n. 5, p. 203-208, 2006. Disponível em: <http://www.sciencedirect.com/science/article/pii/S147149220 6000596>. Acesso em: 17 set. 2013. doi: http://dx.doi. org/10.1016/j.pt.2006.02.015.

SHARMA, P.; SHARMA, A.; SEHGAL, R.; MALLA, N.; KHURANA, S. Genetic diversity of Cryptosporidium isolates from patients in North India. International Journal of Infectious Diseases, v. 17, n. 8, p. 601-605, 2013. Disponível em: <http://www. sciencedirect.com/science/article/pii/S1201971212013239>. Acesso em: 14 set. 2013. doi: http://dx.doi.org/10.1016/j. ijid.2012.12.003.

SMITH, H. V.; NICHOLS, R. A. B. Cryptosporidium: detection in water and food. Experimental Parasitology, v. 124, n. 1, p. 61-79, 2010. SOUZA, P. N. B.; BOMFIM, T. C.; ABBOUD, L. C.; GOMES, R. S. Natural infection by Cryptosporidium sp., Giardia sp. and Eimeria leuckarti in three groups of equines with different handlings in Rio de Janeiro, Brazil. Veterinary Parasitology, v. 160, n. 3-4, p. 327-333, 2009. Disponível em: <http://www.sciencedirect.com/ science/article/pii/S030440170800633X $>$. Acesso em: 13 out. 2013. doi: http://dx.doi.org/10.1016/j.vetpar.2008.10.103.

TOSCAN, G.; PEREIRA, R. C. F.; ARAUJO, L.; SANGIONI, L. A.; VOGEL, F. S. F. Comparação da prevalência de Cryptosporidium spp. em equinos de tração e em atletas do Jockey Club de Santa Maria, RS, Brasil. Ciência Animal Brasileira, v. 11, n. 2, p. 436-440, 2010. WIDMER, G.; YONGSUN, L.; HUNT, P.; MARTINELLI, A.; TOLKOFF, M.; BODI, K. Comparative genome analysis of two Cryptosporidium parvum isolates with different host range. Infection, Genetics and Evolution, v. 12, n. 6, p. 1213-1221, 2012. Disponível em: <http://www.sciencedirect.com/science/article/ pii/S1567134812001086>. Acesso em: 25 out. 2013. doi: http:// dx.doi.org/10.1016/j.meegid.2012.03.027. 